\title{
Superconducting Magnets for LHC Insertions
}

\author{
R. Ostojic
}

\begin{abstract}
The LHC comprises eight insertions, four of which are dedicated to the experiments while the others are used for major collider systems. The various functions of the insertions are fulfilled by a variety of magnet systems, most of them based on the technology of NbTi superconductors cooled by superfluid helium at $1.9 \mathrm{~K}$. In this paper, we review the concepts underlying the design of the LHC insertions, and describe the corresponding design of the various specialized magnet systems. A status of the procurement of the magnets is given, and plans for their installation and commissioning reviewed.
\end{abstract}

Index Terms-Insertions, LHC, quadrupoles, superconducting.

\section{INTRODUCTION}

$\mathbf{T}$ HE Large Hadron Collider, under construction in CERN, is designed to provide proton-proton collisions at center-of-mass energy of $14 \mathrm{TeV}$ and luminosity of $10^{34} \mathrm{~cm}^{-2} \mathrm{~s}^{-1}$. The LHC consists of two interleaved rings, whose main elements are the two-in-one superconducting dipoles and quadrupoles operating in superfluid helium at $1.9 \mathrm{~K}$. The collider will be installed in the existing tunnel with a circumference of $26.7 \mathrm{~km}$, which housed the LEP collider. The layout of the machine closely mirrors that of LEP, with eight identical arcs, each $2.8 \mathrm{~km}$ long, separated by eight $540 \mathrm{~m}$ long straight sections. Two diametrically opposite insertions in Points 1 and 5 house the high-luminosity multipurpose experiments ATLAS and CMS, for which considerable civil engineering was required. The more specialized experiments ALICE and $\mathrm{LHCb}$ are installed in the existing caverns in Points 2 and 8 . These insertions are also equipped with beam injection systems for the two rings. The remaining four insertions house other major collider systems: Points 3 and 7 are dedicated to beam collimation, while Points 4 and 6 serve respectively for the RF and beam extraction.

The optics and layout of the LHC insertions have been optimized with respect to their function and technical and infrastructure constraints specific to each tunnel area. Although the general layout of the LHC has not been modified since 1995 [1], the layout of the insertions has been regularly updated. As an example, the CMS high-luminosity insertion has been modified to provide the necessary tuning flexibility for the TOTEM small-angle experiment, and similar features have been implemented in the ATLAS insertion. The collimation systems in Points 3 and 7, designed to limit the beam losses in the superconducting magnets and the background in the experiments, have been considerably reworked to increase their efficiency.

Manuscript received October 20, 2003

The author is with the Accelerator Technology Division, CERN, Geneva, Switzerland (e-mail: Ranko.Ostojic@cern.ch).

Digital Object Identifier 10.1109/TASC.2004.829040
Similarly, Point 4 was rearranged and greatly simplified by displacing the superconducting RF cavities to the existing experimental cavern, and reducing the number of quadrupoles and separation dipoles. All these modifications were necessary to resolve the functional or installation bottlenecks that were identified with the advance of equipment design and its integration in the tunnel.

In spite of functional differences, all insertions comprise, Fig. 1, moving from the arc toward the middle of an insertion, a dispersion suppressor followed by a matching section, a section of separation dipoles, and in the experimental insertions, the final focus system (low- $\beta$ triplet). Most of the magnets in the insertions are based on the state-of-the-art superconducting magnet technology of NbTi superconductors cooled by superfluid helium at $1.9 \mathrm{~K}$. However, a number of stand-alone quadrupoles in the matching sections, as well as the separation dipoles, are operated at $4.5 \mathrm{~K}$. Furthermore, in the high radiation areas specialized normal conducting magnets are used. The areas in between the magnets house the beam injection and extraction systems, collimators and the RF system and beam instrumentation.

A significant part of the magnet systems in the insertions is supplied by accelerator laboratories world-wide as part of the contributions of respective nonmember countries to the LHC. In view of the complicated interfaces, advanced stage of equipment design and tight budgets, close collaboration and frequent contacts have been established between laboratories, which have allowed efficient monitoring of progress and close configuration control.

Following extensive R\&D, industrial contracts for the procurement of the superconducting magnets for the LHC insertions have been awarded, or their construction started in laboratory production facilities. Although production of some magnets is nearing completion and shipments to CERN have started, most of the magnets will be delivered during the next two years. Installation of the first LHC octant (Octant 7-8) is planned for mid 2004. Following its commissioning, a major milestone for the project is the octant injection test, planned for spring 2006. In this report, we review the status of the various superconducting magnet systems, and report on the installation and commissioning plans.

\section{The Final Focus Magnets}

The low- $\beta$ triplet, Fig. 2 , is located at $23 \mathrm{~m}$ on each side of the interaction point, and is composed of four single aperture quadrupoles with a coil aperture of $70 \mathrm{~mm}$. It is cooled with superfluid helium at $1.9 \mathrm{~K}$ using an external heat exchanger system capable of extracting up to $10 \mathrm{~W} / \mathrm{m}$ of power deposited in the coils by the secondaries emanating from the p-p collisions. Two types of quadrupoles are used in the triplet, $6.6 \mathrm{~m}$ long MQXA 


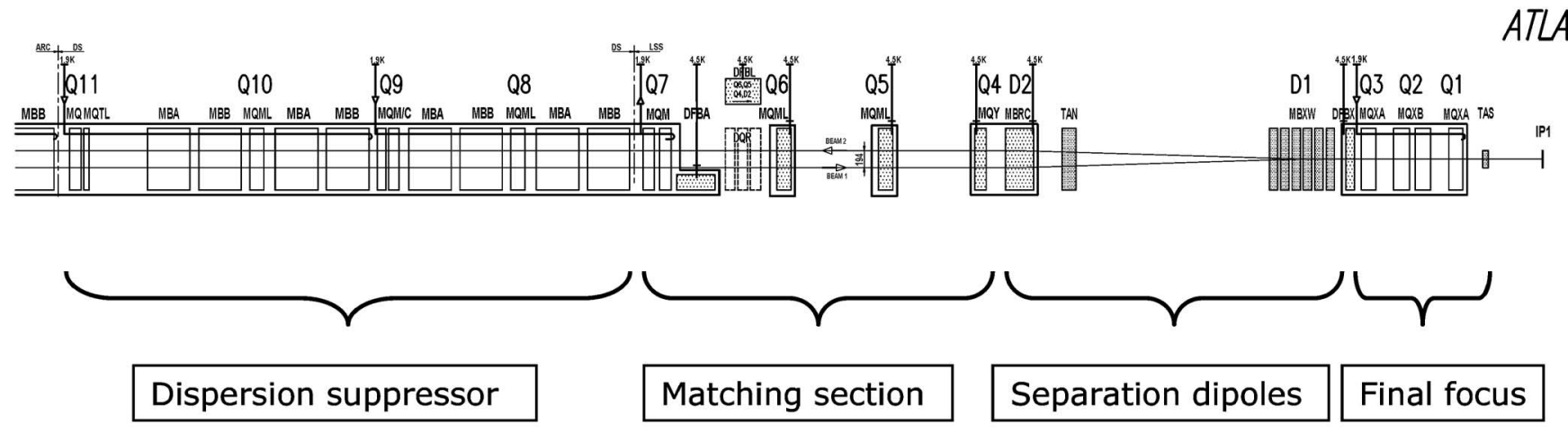

Fig. 1. Schematic layout of the ATLAS experimental insertion.

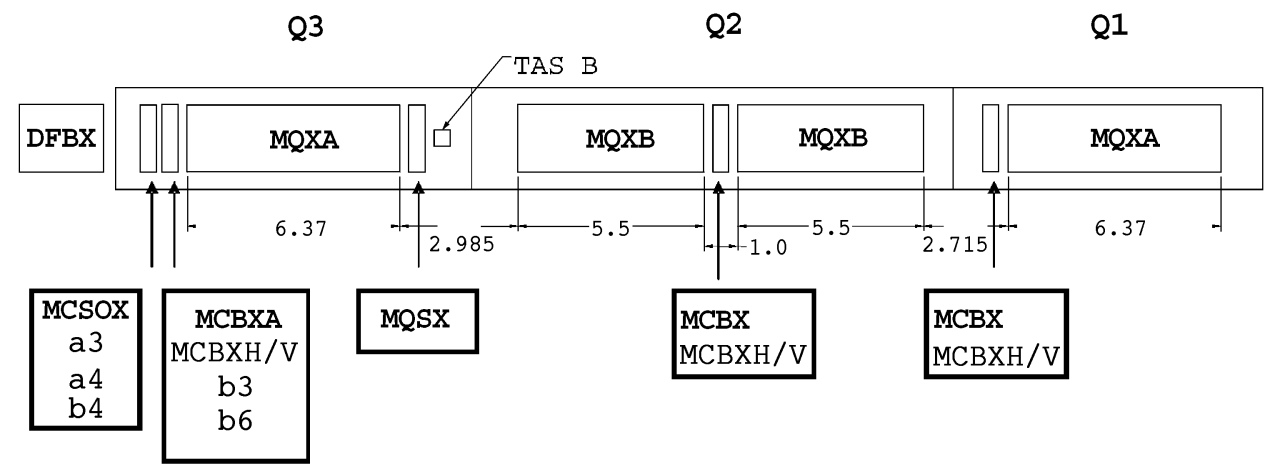

Fig. 2. Schematic layout of the LHC low- $\beta$ triplet.

magnets designed and developed by KEK (Japan), and $5.7 \mathrm{~m}$ long MQXB magnets designed and built by FNAL (USA). The magnets are powered in series with $7 \mathrm{kA}$, with an additional inner loop of $5 \mathrm{kA}$ for the MQXB magnets. Together with the orbit and multipole correctors supplied by CERN, the low- $\beta$ quadrupoles are completed in their cold masses and cryostated by FNAL. The cryogenic feedboxes, providing a link to the cryogenic distribution line and power converters, are designed and built by LBNL (USA) [2].

\section{A. Low- $\beta$ Quadrupoles}

Alongside the LHC main dipoles, the high-gradient, wideaperture low- $\beta$ quadrupoles are the most demanding magnets in the collider. They must operate reliably at $215 \mathrm{~T} / \mathrm{m}$, sustain extremely high heat loads in the coils and radiation dose during their lifetime, and have a very good field quality within the $63 \mathrm{~mm}$ aperture of the cold bore. The design of the MQXA quadrupole is based on a four-layer coil using $11 \mathrm{~mm}$ wide Rutherford-type graded NbTi cables [3]. The coils are wound and cured in two double layers, and are assembled using $10 \mathrm{~mm}$ wide spacer-type collars. The pre-stress in the coils and their rigidity is provided by the yoke structure, which consists of horizontally split laminations keyed at the mid-plane. The main parameters of the magnet are given in Table I.

Following a comprehensive R\&D and prototype program, KEK has entrusted the fabrication of the MQXA quadrupoles to Toshiba Corporation [4]. The final testing and field measurements are performed in the vertical testing facility in KEK, Fig. 3. To date, 15 magnets out of the series of 18 were produced and 13 tested. The fabrication of the remaining four
TABLE I

MAIN PARAMETERS OF THE MQXA LOW- $\beta$ QUADRUPOLE

\begin{tabular}{lcc}
\hline \hline Coil inner diameter & \multicolumn{3}{c}{$70 \mathrm{~mm}$} \\
Magnetic length & \multicolumn{3}{c}{$6.37 \mathrm{~m}$} \\
Operating temperature & \multicolumn{3}{c}{$1.9 \mathrm{~K}$} \\
Nominal gradient & \multicolumn{3}{c}{$215 \mathrm{~T} / \mathrm{m}$} \\
Nominal current & \multicolumn{3}{c}{$7149 \mathrm{~A}$} \\
Peak field in coil & \multicolumn{3}{c}{$8.6 \mathrm{~T}$} \\
Quench field & \multicolumn{3}{c}{$10.7 \mathrm{~T}$} \\
Stored energy per aperture & \multicolumn{3}{c}{$2300 \mathrm{~kJ}$} \\
Inductance per aperture & \multicolumn{3}{c}{$90.1 \mathrm{mH}$} \\
Cable parameters & Cable 1 & Cable 2 \\
Width (mm) & 11 & 11 \\
Mid-thickness (mm) & 1.487 & \multicolumn{2}{c}{1.340} \\
Keystone angle (deg.) & 2.309 & 1.319 \\
No of strands & 27 & 30 \\
Strand dia. (mm) & 0.815 & 0.735 \\
Cu/SC Ratio & 1.2 & 1.9 \\
Filament dia. $(\mu \mathrm{m})$ & 10 & 10 \\
$\mathrm{j}_{\mathrm{c}}\left(\mathrm{A} / \mathrm{mm}^{2}, 4.2 \mathrm{~K}\right.$ and $\left.6 \mathrm{~T}\right)$ & 2200 & 2160 \\
\hline \hline
\end{tabular}

magnets will be completed in the beginning of 2004, and all magnets delivered to Fermilab for further assembly by end of 2004.

The MQXB design features a two-layer coil, with each layer individually wound using a $15.4 \mathrm{~mm}$ wide Rutherford-type NbTi cable [5]. The coils are assembled using free-standing collars, which provide the pre-stress and react the magnetic forces. The collared assembly is aligned in the yoke structure with precision keys, and the magnet enclosed in a stainless steel helium vessel consisting of half-shells welded at the pole plane. The design parameters of the magnet are given in Table II. 


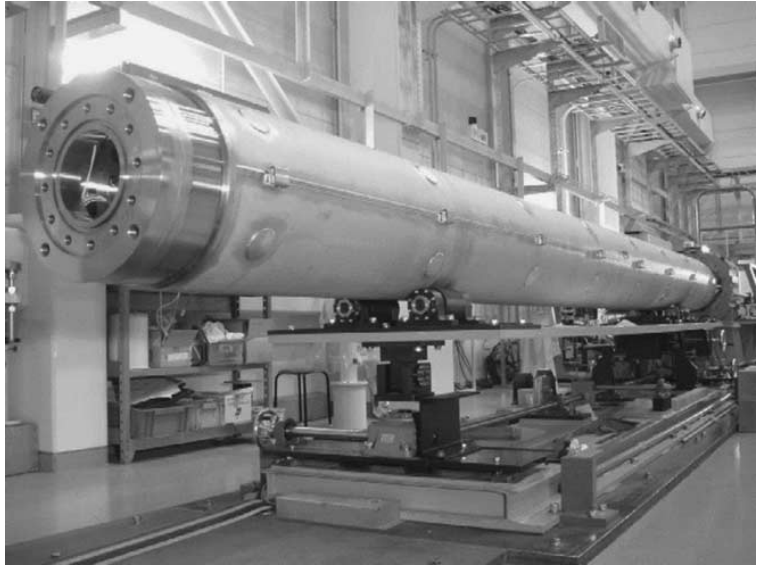

Fig. 3. MQXA low- $\beta$ quadrupole on the alignment bench in KEK.

The MQXB design was validated in an extensive program of short models and full-length prototypes, following which the series production has started in FNAL [6]. In Fig. 4, the first Q2 assembly, comprising two MQXB series quadrupoles is shown during testing in the horizontal test facility in FNAL. To date, eleven of the total of $18 \mathrm{MQXB}$ have been completed, and two others are in different phases of manufacturing and testing.

Power testing of both MQXA and MQXB low- $\beta$ quadruples has in general shown good training behavior. All magnets reached $230 \mathrm{~T} / \mathrm{m}$ after relatively short training cycles, confirmed after another cool-down. In view of this experience, a thermal cycle is now considered unnecessary for the remaining magnets. As an exception to the rule, KEK has observed an increase in the number of training quenches in the recent MQXA production, while the MQXB04 magnet did not reach the nominal gradient, most likely due to cable damage. On the other hand, all magnets showed very good and stable field quality, which is also of primary importance for achieving the nominal performance of the LHC. The first complete triplet is expected at CERN by mid-2004.

\section{B. Correctors}

All three quadrupole assemblies in the inner triplet are equipped with several different types of correctors supplied by CERN [7]. As the MCBX orbit correctors are part of the Q2 assembly, Fig. 5, their timely delivery is of prime importance for the production and tests of the MQXB magnets. CERN has adjudicated the contract for fabrication of the dipole correctors to SigmaPhi (France). Due to technical difficulties encountered in the pre-series production, delivery of these magnets has been delayed. In order to speed up deliveries, CERN has taken over the responsibility for their testing. It is expected that the present production and test cycle of two dipole correctors per month will allow completion and delivery of all triplet quadrupoles by September 2005 as initially planned.

\section{SePARATION Dipoles}

The separation dipoles are used to change the beam separation in the insertions from the nominal $194 \mathrm{~mm}$ in the LHC arcs. In the experimental insertions the pair of D1-D2 dipoles
TABLE II

MAIN PARAMETERS OF THE MQXB LOW- $\beta$ QUADRUPOLE

\begin{tabular}{|c|c|c|}
\hline Coil inner diameter & \multicolumn{2}{|c|}{$70 \mathrm{~mm}$} \\
\hline Magnetic length & \multicolumn{2}{|c|}{$5.5 \mathrm{~m}$} \\
\hline Operating temperature & \multicolumn{2}{|c|}{$1.9 \mathrm{~K}$} \\
\hline Nominal gradient & \multicolumn{2}{|c|}{$215 \mathrm{~T} / \mathrm{m}$} \\
\hline Nominal current & \multicolumn{2}{|c|}{$11950 \mathrm{~A}$} \\
\hline Peak field in coil & \multicolumn{2}{|c|}{$7.7 \mathrm{~T}$} \\
\hline Quench field & \multicolumn{2}{|c|}{$9.2 \mathrm{~T}$} \\
\hline Stored energy per aperture & \multicolumn{2}{|c|}{$1360 \mathrm{~kJ}$} \\
\hline Inductance per aperture & \multicolumn{2}{|c|}{$19.1 \mathrm{mH}$} \\
\hline Cable parameters & Cable 1 & Cable 2 \\
\hline Width (mm) & 15.4 & 15.4 \\
\hline Mid-thickness (mm) & 1.456 & 1.146 \\
\hline Keystone angle (deg.) & 1.079 & 0.707 \\
\hline No of strands & 37 & 46 \\
\hline Strand dia. $(\mathrm{mm})$ & 0.808 & 0.650 \\
\hline $\mathrm{Cu} / \mathrm{SC}$ Ratio & 1.3 & 1.8 \\
\hline Filament dia. $(\mu \mathrm{m})$ & 6 & 6 \\
\hline $\mathrm{j}_{\mathrm{c}}\left(\mathrm{A} / \mathrm{mm}^{2}, 4.2 \mathrm{~K}\right.$ and $\left.5 \mathrm{~T}\right)$ & 2750 & 2750 \\
\hline
\end{tabular}

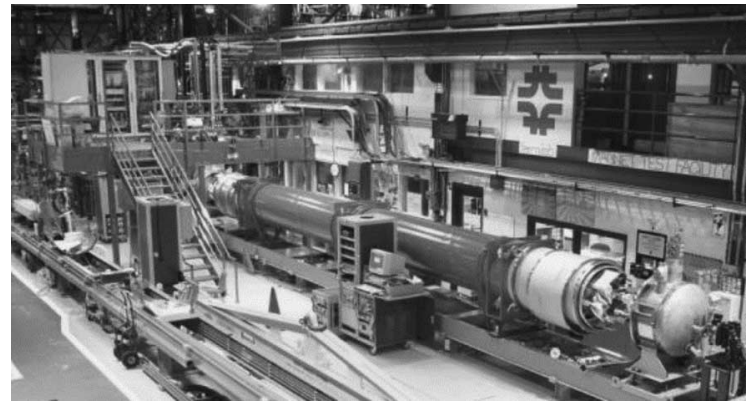

Fig. 4. First Q2 low- $\beta$ quadrupole on the test stand in Fermilab.

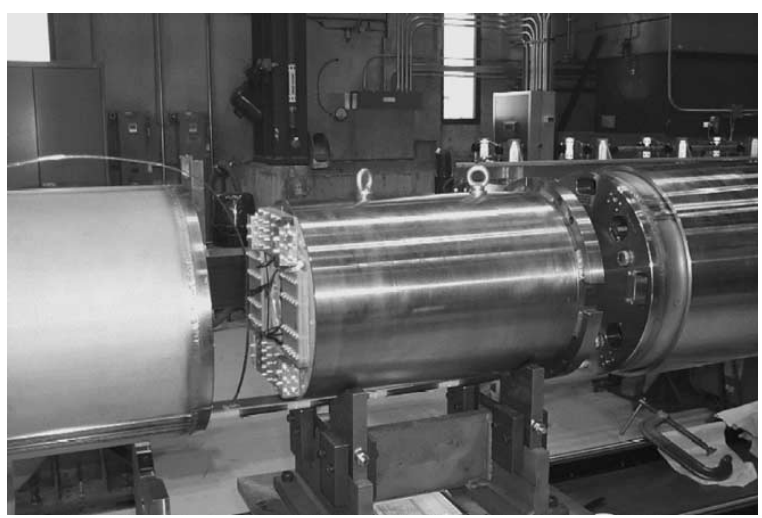

Fig. 5. Mounting of the MCBX dipole corrector between two MQXB quadrupoles in the first Q2 magnet.

brings the two beams onto a colliding orbit at the interaction points. To reduce the beam-beam effects, the first separation dipole D1 is placed immediately upstream of the low- $\beta$ triplet. In the high-luminosity insertions, high radiation levels are expected and normal conducting magnets are used. In the ALICE and $\mathrm{LHCb}$ insertions, D1 is a stronger superconducting magnet (MBX) allowing more space for the injection systems. In all cases, the D2 separation dipole (MBR) is a twin-aperture superconducting magnet. In the RF insertion, a beam separation of $420 \mathrm{~mm}$ is required for the superconducting cavities, and superconducting dipoles MBR and MBRS are installed for reasons of space limitation. 
TABLE III

MAIN PARAMETERS OF THE MBX, MBR AND MBRS SUPERCONDUCTING SEPARATION DIPOLES

\begin{tabular}{lc}
\hline Coil inner diameter & $80 \mathrm{~mm}$ \\
Magnetic length & $9.45 \mathrm{~m}$ \\
Operating temperature & $1.9 \mathrm{~K}(\mathrm{MBX})$ \\
& $4.5 \mathrm{~K}(\mathrm{MBR}, \mathrm{MBRS})$ \\
Aperture separation & $-(\mathrm{MBX})$ \\
& $188 / 194 \mathrm{~mm}(\mathrm{MBR})$ \\
& $416 \mathrm{~mm}(\mathrm{MBRS})$ \\
Nominal field & $3.8 \mathrm{~T}$ \\
Nominal current & $5750 \mathrm{~A}(\mathrm{MBX}, \mathrm{MBRS})$ \\
& $6050 \mathrm{~A} \mathrm{(MBR})$ \\
Peak field in coil & $4.2 \mathrm{~T}$ \\
Quench field & $4.8 \mathrm{~T}$ \\
Stored energy per aperture & $470 \mathrm{~kJ}$ \\
Inductance per aperture & $25.8 \mathrm{mH}$ \\
Cable parameters & \\
Width (mm) & $9.73 \mathrm{~mm}$ \\
Mid-thickness & $1.166 \mathrm{~mm}$ \\
Keystone angle & $1.2 \mathrm{deg}$. \\
No of strands & 30 \\
Strand dia. & $0.648 \mathrm{~mm}$ \\
Cu/SC Ratio & 1.8 \\
Filament dia. & $6 \mu \mathrm{m}$ \\
jc (A/mm², 4.2 K and 5 T) & 2500 \\
\hline \hline
\end{tabular}

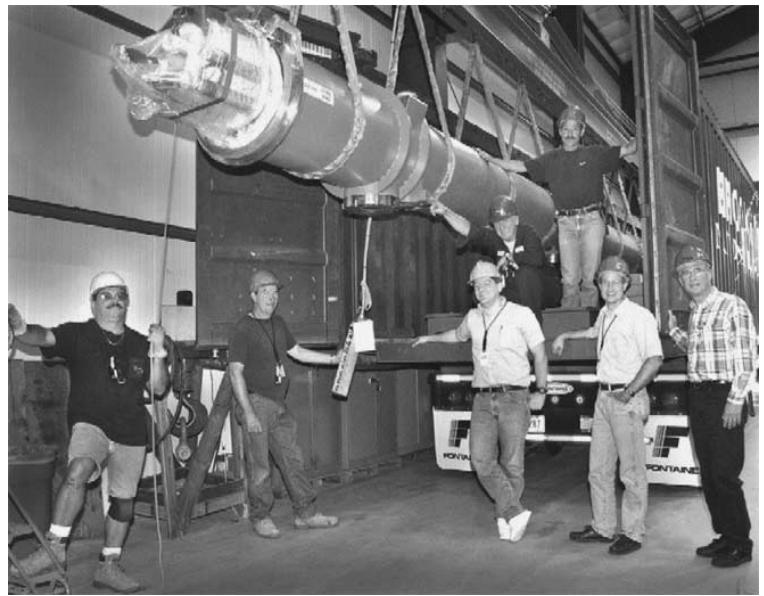

Fig. 6. The first D1 separation dipole loaded in the container in BNL for shipment to CERN.

The MBX, MBR and MBRS dipoles are designed and built by BNL (USA) on the basis of the RHIC lattice dipole, and feature a $10 \mathrm{~m}$ long, $80 \mathrm{~mm}$ aperture coil. While MBX and MBRS are single aperture magnets, essentially identical to the RHIC lattice dipole (except for the sagitta and cooling method), MBR is a two-in-one magnet which considerably deviates from the RHIC design [8]. All magnets operate at a field of up to $3.8 \mathrm{~T}$, and their main parameters are given in Table III.

BNL has practically completed the fabrication of all twenty separation dipoles. The remaining D3 magnets are in an advanced stage of completion. The magnets were cold tested in the horizontal test facility in BNL, after which they were prepared for shipping to CERN. Four MBX dipoles have already been delivered, Fig. 6, and it is expected that the D2 magnets, Fig. 7, will follow soon. The shipping to CERN of the remaining magnets is expected by the mid 2005 .

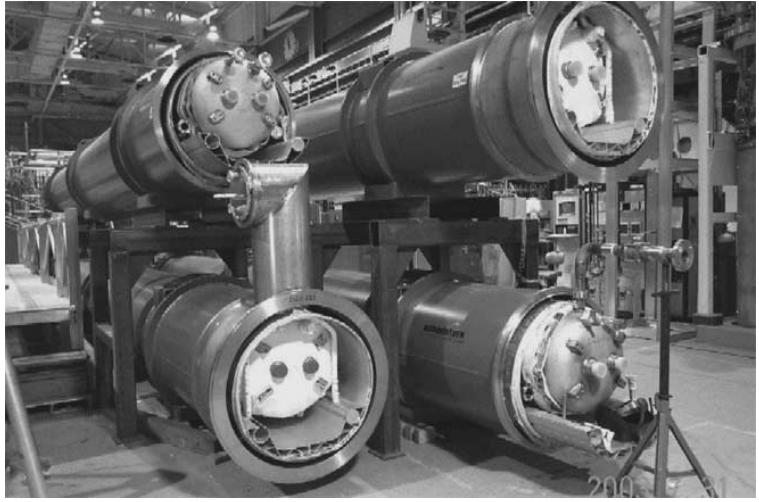

Fig. 7. After testing, D2 magnets are completed in BNL with the "jumper" modules for connection to the LHC cryo-line and stored for shipment.

\section{DISPERSION SUPPRESSORS AND MATCHING SECTIONS}

The optics flexibility of the LHC insertions is provided by the individually powered quadrupoles in the dispersion suppressors and the matching sections. All dispersion suppressors comprise four half cells, with two main LHC dipoles per half cell. The suppressors are an integral part of the arc cryostat string, and have identical powering and cryogenic infrastructure. The matching sections consist of stand alone quadrupoles, but the number and parameters of the magnets are specific for each insertion. They also comprise four half cells, except in Points 4 and 6 , where the matching section has respectively 3 and 2 quadrupoles. In the cleaning insertions, specialized normal conducting quadrupoles are used in the part of the matching section located in high radiation areas.

CERN has developed two types of superconducting quadrupoles for the dispersion suppressors and matching sections, one featuring a $56 \mathrm{~mm}$ aperture coil (MQM) [9], and the other an enlarged, $70 \mathrm{~mm}$ aperture coil (MQY) [10]. Both quadrupoles use narrow cables, so that the nominal current is less than $6 \mathrm{kA}$, substantially simplifying the warm and cold powering circuits.

The MQM quadrupole consists of two identical, independently powered apertures, which are assembled together in a common yoke structure. The coils are made using an $8.8 \mathrm{~mm}$ wide Rutherford-type NbTi cable wound as a double layer and cured in a single cycle. The design was optimized for the highest transfer function and operational margin. The coils are assembled using $21 \mathrm{~mm}$ wide collars, locked with four full-length tapered keys, which provide the necessary compressive stress in the coils and withstand the magnetic forces. The collared apertures are assembled together using two-in-one iron laminations, similar to the LHC arc quadrupole, so that the cooling and bus bar systems in the dispersion suppressors are fully compatible. Three versions of the MQM quadrupole are required for the LHC, with magnetic lengths of $2.4 \mathrm{~m}, 3.4 \mathrm{~m}$ and $4.8 \mathrm{~m}$. The main parameters of the quadrupole are listed in Table IV.

Following the development work in CERN, the contract for the supply of $96 \mathrm{MQM}$ quadrupoles was adjudicated to Tesla Engineering (UK). During 2003, a pre-series of four MQM magnets have been completed and tested in the $1.9 \mathrm{~K}$ vertical test facility at CERN, Fig. 8. All magnets achieved the ultimate operating gradient in the LHC of $216 \mathrm{~T} / \mathrm{m}$, with a minimal 
TABLE IV

MAIN PARAMETERS OF THE MQM QUADRUPOLE

\begin{tabular}{lc}
\hline \hline Coil inner diameter & $56 \mathrm{~mm}$ \\
Magnetic length & $2.4 \mathrm{~m}, 3.4 \mathrm{~m}, 4.8 \mathrm{~m}$ \\
Operating temperature & $1.9 \mathrm{~K}$ \\
Nominal gradient & $200 \mathrm{~T} / \mathrm{m}$ \\
Nominal current & $5390 \mathrm{~A}$ \\
Peak field in coil & $6.13 \mathrm{~T}$ \\
Quench field & $7.58 \mathrm{~T}$ \\
Stored energy per aperture & $64.3 \mathrm{~kJ} / \mathrm{m}$ \\
Inductance per aperture & $4.44 \mathrm{mH} / \mathrm{m}$ \\
Cable parameters & \\
Width & $8.8 \mathrm{~mm}$ \\
Mid-thickness & $0.845 \mathrm{~mm}$ \\
Keystone angle & $0.91 \mathrm{deg}$. \\
No of strands & 36 \\
Strand dia. & $0.475 \mathrm{~mm}$ \\
Cu/SC Ratio & 1.75 \\
Filament dia. & $6 \mu \mathrm{m}$ \\
$\mathrm{j}_{\mathrm{c}}\left(\mathrm{A} / \mathrm{mm}^{2}, 4.2 \mathrm{~K}\right.$ and $\left.5 \mathrm{~T}\right)$ & 2800 \\
\hline \hline
\end{tabular}

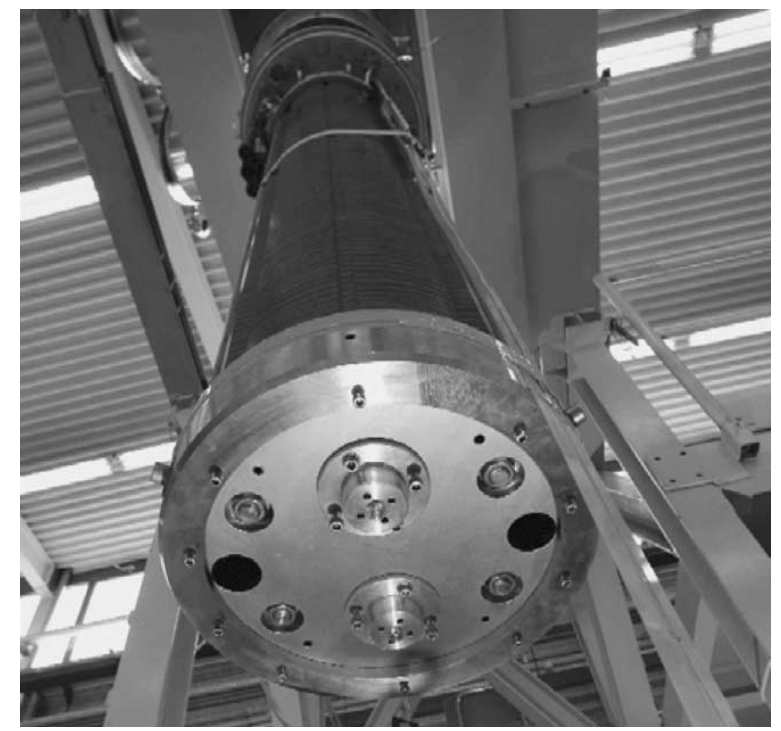

Fig. 8. The pre-series MQM quadrupole in the $1.9 \mathrm{~K}$ vertical test station at CERN.

number of training quenches [11]. Their performance in terms of field quality is also satisfactory. These encouraging results confirmed the robustness of the design and the quality of the production techniques. The pre-series phase has also shown the importance of close quality control during production for successful performance of the magnets. The contractor is now ramping-up the fabrication, with the goal of producing four MQM magnets per month.

Similarly to MQM, the MQY wide-aperture quadrupole consists of two $3.6 \mathrm{~m}$ long individually powered apertures assembled in a common yoke structure. The $70 \mathrm{~mm}$ aperture coils have four layers. The inner two layers are wound and cured in a single cycle using two $8.3 \mathrm{~mm}$ wide Rutherford-type graded $\mathrm{NbTi}$ cables, with the transition between the cables in the middle of the second layer. The outer two layers are wound and cured using only the smaller cable. The coils are assembled using a self-supporting collar system, consisting of two $22 \mathrm{~mm}$ wide collars which are locked with eight full-length tapered keys. As in the MQM quadrupoles, the collared coils are assembled in
TABLE $\mathrm{V}$

MAIN PARAMETERS OF THE MQY WIDE APERTURE QUADRUPOLE

\begin{tabular}{lcc}
\hline \hline Coil inner diameter & \multicolumn{2}{c}{$70 \mathrm{~mm}$} \\
Magnetic length & \multicolumn{3}{c}{$3.4 \mathrm{~m}$} \\
Operating temperature & \multicolumn{3}{c}{$4.5 \mathrm{~K}$} \\
Nominal gradient & \multicolumn{3}{c}{$160 \mathrm{~T} / \mathrm{m}$} \\
Nominal current & \multicolumn{3}{c}{$3610 \mathrm{~A}$} \\
Peak field in coil & \multicolumn{2}{c}{$6.1 \mathrm{~T}$} \\
Quench field & \multicolumn{2}{c}{$7.5 \mathrm{~T}$} \\
Stored energy per aperture & \multicolumn{2}{c}{$141 \mathrm{~kJ} / \mathrm{m}$} \\
Inductance per aperture & \multicolumn{2}{c}{$21.7 \mathrm{mH} / \mathrm{m}$} \\
Cable parameters & Cable 1 & Cable 2 \\
Width (mm) & 8.3 & \multicolumn{2}{c}{0.3} \\
Mid-thickness (mm) & 1.285 & 0.845 \\
Keystone angle (deg.) & 2.16 & 1.05 \\
No of strands & 22 & 34 \\
Strand dia. (mm) & 0.735 & 0.475 \\
Cu/SC Ratio & 1.25 & 6 \\
Filament dia. $(\mu \mathrm{m})$ & 6 & 6 \\
$\mathrm{j}_{\mathrm{c}}\left(\mathrm{A} / \mathrm{mm}^{2}, 4.2 \mathrm{~K}\right.$ and $\left.5 \mathrm{~T}\right)$ & 2670 & 2800 \\
\hline \hline
\end{tabular}

the two-in-one yoke structure using four full-length keys at the poles, which center the assemblies once the laminations are in place and locked with dowel pins. The main parameters of the quadrupole are given in Table $\mathrm{V}$.

Following the successful model program, the contract for the supply of $26 \mathrm{MQY}$ quadrupoles has been adjudicated to ACCEL Instruments (Germany). The first pre-series magnet has been completed in September 2003 and delivered for qualification tests at CERN [12]. As the contractor is also manufacturing the LHC arc quadrupoles, experienced staff are available for rapid ramping-up of the fabrication.

The quadrupoles for the dispersion suppressors and matching sections comprise several MQM, MQY and dipole corrector magnets and range in length from $5.3 \mathrm{~m}$ to $11.3 \mathrm{~m}$. In spite of the variety of types and interface requirements, the design of the quadrupoles is based on the same principle where two welded half-shells provide the rigidity and alignment of the magnets and serve as a helium pressure vessel. In total, 82 insertion quadrupoles will be assembled in the LHC Magnet Assembly Facility (MAF) at CERN. The tooling and procedures for the cold mass assembly, including electrical connections, longitudinal welds and vessel closure, have been developed and validated on prototype assemblies. The geometrical measurements of the pre-series quadrupole, Fig. 9, have shown that very strict requirements for magnet alignment can be met [13]. The assembly of the dispersion suppressor and matching section quadrupoles is now entering series production.

\section{INSTALLATION AND COMMISSIONING}

Although the number of superconducting magnets in the LHC insertions is small compared to the arcs, the number of units, their strategic position, and in particular the number of different types, requires that full attention be given to the installation and commissioning of these sections of the collider. Integration studies are well underway to determine the infrastructure requirements, and identify any interference with other equipment in their final position and during transport. Due to their nonstandard overall dimensions, studies of special handling devices and routing directions have also been started. 


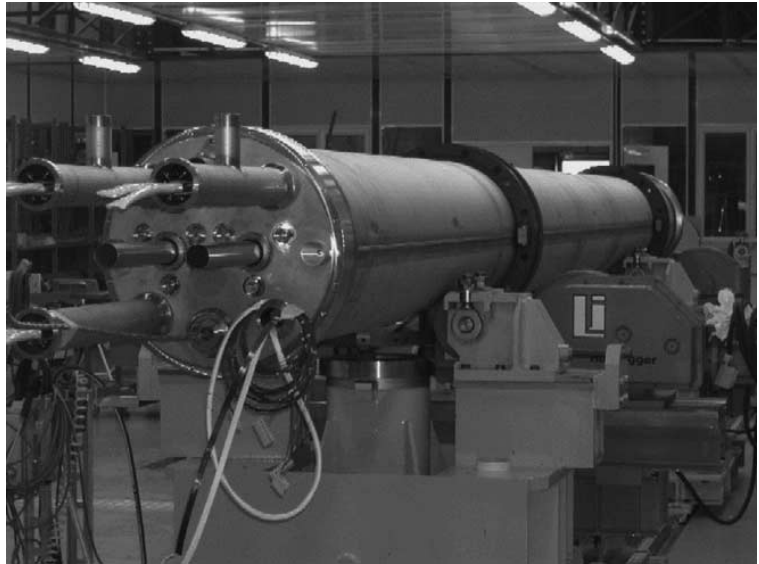

Fig. 9. The first dispersion suppressor quadrupole Q9 on the test stand for alignment measurements at CERN.

The updated installation schedule of the LHC foresees the start of installation of Octant 7-8 in April 2004 and its commissioning in the period April-September 2005. This octant comprises the noninjection side of the experimental insertion in Point $8(\mathrm{LHCb})$ and also part of the cleaning insertion in Point 7. As such, all types of insertion magnets are concerned by this first commissioning activity. Furthermore, the beam injection test, a very important milestone for the LHC foreseen for April 2006, requires that the injection side of Point 8 is also commissioned. In view of the tight schedule, detailed planning for the hardware commissioning is now in elaboration. The basis of these plans is the experience gained in commissioning and running the LHC String. However, to be most effective, involvement in commissioning of laboratories which manufactured and tested the insertion magnets, in particular the low- $\beta$ triplets, is also expected and appropriate preparations are in progress.

\section{CONCLUSION}

Following comprehensive $R \& D$ and prototype programs, the fabrication of the superconducting magnets for LHC insertions has now entered a decisive stage of ramping-up the production to full speed. The experience from pre-series production has shown that although the industry is in general well prepared, the key for achieving stringent performance of the LHC magnets remains adherence to strict quality control rules.
A significant number of superconducting magnets for the LHC insertions are produced in accelerator laboratories in Japan and USA, as part of the collaboration agreements with nonmember states. Excellent and constructive relations have been established with the teams involved. In most cases, the production is nearing completion, and final deliveries are foreseen by the end of 2005 .

\section{ACKNOWLEDGMENT}

The work reported in this paper is the result of the technical expertise, ingenuity and dedication of a number of teams in CERN, in the collaborating laboratories world-wide and in industry. The author wishes to acknowledge their excellent results, and is grateful for the contributions received.

\section{REFERENCES}

[1] The Large Hadron Collider.

[2] J. S. Kerby, "Production status of the LHC inner triplet magnet system," IEEE Trans. Appl. Supercond., vol. 13, no. 2, pp. 1229-1234, June 2003.

[3] K. Tsuchiya et al., "Magnetic design of a low-beta quadrupole magnet for the Large Hadron Collider," IEEE Trans. Appl. Supercond., vol. 10, no. 1, pp. 135-138, March 2000.

[4] T. Nakamoto et al., "Production and performance of the LHC interaction region quadrupoles at KEK," IEEE Trans. Appl. Supercond., vol. 13, no. 2, pp. 1321-1324, June 2003.

[5] R. Bossert et al., "Development of a high gradient quadrupole for the LHC interaction regions," IEEE Trans. Appl. Supercond., vol. 7, no. 1, pp. 751-754, March 1997.

[6] - "Construction experience with MQXB quadrupole magnets built at Fermimlab for the LHC interaction regions," IEEE Trans. Appl. Supercond., vol. 13, no. 2, pp. 1297-1300, June 2003.

[7] R. Wolf, "Status of the Production of the Corrector Magnets for the LHC,", this conference.

[8] E. Willen et al., "Superconducting dipole magnets for the LHC insertion regions," in Proc. EPAC'2000, Vienna, Austria, June 2000, pp. 2187-2189.

[9] J. Lucas et al., "Performance of the single and twin-aperture models of the $6 \mathrm{kA}$ superconducting quadrupole for the LHC insertions," IEEE Trans. Appl. Supercond., vol. 11, no. 1, pp. 1645-1648, March 2001.

[10] G. Kirby et al., "Performance of the $1-\mathrm{m}$ model of the $70 \mathrm{~mm}$ bore twin-aperture quadrupole for the LHC insertions," IEEE Trans. Appl. Supercond., vol. 11, no. 1, pp. 1641-1644, March 2001.

[11] R. Ostojic et al., "Construction and Qualification of the Pre-Series MQM-Type Superconducting Quadrupoles for the LHC Insertions,", this conference.

[12] G. Kirby et al., "Fabrication of the Pre-Series Wide Aperture Superconducting Quadrupoles for the LHC Insertions,", this conference.

[13] R. Ostojic et al., "Design, Assembly and Mechanical Behavior of the Pre-Series LHC Insertion Quadrupoles,", this conference. 